REVISTA CIENCIAS BIOMÉDICAS

\title{
PRESENTACIÓN DE CASOS CLÍNICOS
}

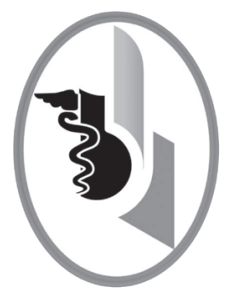

\section{EVALUACIÓN GAMMAGRAFICA DE GLÁNDULAS SALIVALES EN SÍNDROME DE SJÖGREN}

\author{
SALIVARY GLAND SCINTIGRAPHY IN SJÖGREN SYNDROME
}

\author{
Betancourt Piñeres Aiken Felipe ${ }^{1}$ \\ Milanés Pérez Rosa Isabel² \\ Bonnet Palencia Igor Iván ${ }^{3}$ \\ Padilla Tovar Lacides ${ }^{4}$ \\ Moreno Grau Álvaro ${ }^{4}$ \\ Álvarez Romero Erick ${ }^{5}$ \\ Muñoz Méndez José Thomas ${ }^{5}$ \\ Correspondencia: aikenbet@hotmail.com \\ Recibido para evaluación: septiembre - 20 - 2010. Aceptado para publicación: febrero - 10 - 2011
}

\section{RESUMEN}

El Síndrome de Sjögren es una enfermedad sistémica autoinmune que afecta principalmente las glándulas exocrinas del organismo, como las salivales y las lacrimales provocando una disminución de sus secreciones, por lo que también se denomina síndrome seco. Se presenta caso clínico de una paciente de sexo femenino la cual cumple todos los criterios clasificatorios del Consenso Europeo Americano 2002 para el Síndrome de Sjogren. Rev.cienc.biomed. 2011; 2(1): 130-134

\section{PALABRAS CLAVES}

Síndrome de Sjögren. Glándulas salivales. Gammagrafía.

\section{SUMMARY}

Sjögren syndrome is a systemic autoimmune disease that mainly affects exocrine glands, such as the salivary and lacrimal glands, causing a decline in their secretions. This disease is also known as Sicca Syndrome. It is reported a clinical case of a woman who fulfill all diagnostic criteria of European-American Consensus of Sjogren Syndrome 2002. Rev.cienc.biomed. 2011; 2(1): 130-134

\section{KEYWORDS}

Sjogren syndrome. Scintigraphy. Salivary glands.

1 Médico. Especialista en Medicina Nuclear. Docente Departamento de Diagnóstico. Facultad de Medicina, Universidad de Cartagena. Colombia.

2 Médico. Especialista en Otorrinolaringología. Docente Departamento Quirúrgico. Facultad de Medicina. Universidad de Cartagena. Colombia.

3 Médico. Especialista en Medicina Nuclear. Nucleodiagnóstico Ltda. Cartagena. Colombia.

4 Médico. Especialista en Medicina Interna y Reumatología. Docente Departamento Médico. Facultad de Medicina. Universidad de Cartagena. Colombia.

5 Estudiante Facultad de Medicina. Universidad de Cartagena. Colombia. 
Betancourt Piñeres Aiken Felipe, Milanés Pérez Rosa Isabel, Bonnet Palencia Igor Iván, Padilla Tovar Lacides,

Moreno Grau Álvaro, Álvarez Romero Erick, Muñoz Méndez José Thomas

\section{INTRODUCCIÓN}

El Síndrome de Sjögren es un trastorno auto inmunitario crónico de etiología desconocida, que se caracteriza por infiltración linfoplasmocitaria de las glándulas exocrinas con destrucción epitelial, provocando una hipofunción de las mismas y por ende un síndrome seco definido por sequedad oral (xerostomía) y ocular (xeroftalmía) (1).

Existen dos formas clínico-patológicas de Síndrome de Sjögren. Primaria o síndrome seco, que cursa aisladamente. La secundaria es asociada a otra conectivopatia y con más frecuencia a la Artritis Reumatoide, como también al Lupus, Esclerosis sistémica, Polimiositis, Hepatitis autoinmune, Cirrosis biliar, entre otras $(2,3,4,5,6,7)$.

Es más frecuente en mujeres (9:1) y su prevalencia se encuentra en torno al 2,7\%. Afecta principalmente a personas en edades de 40-60 años. Al no conocerse la causa de esta enfermedad, el diagnóstico se basa principalmente en los datos clínicos y analíticos.

En 1996, Vitali et al (8), propusieron los criterios europeos, que tienen en cuenta tanto la sintomatología bucal como sus signos de afectación mediante pruebas complementarias y de laboratorio, en donde la gammagrafía salival tiene gran protagonismo. La sensibilidad y especificidad de la gammagrafía se considera del $87,2 \%$ y $79 \%$ respectivamente.

Posteriormente se realizó un Consenso Europeo-Americano el cual modificó los criterios propuestos por Vitali et al., expresados en la tabla No 1, siendo necesarios por lo menos cuatro de ellos para confirmar el diagnóstico (8).

\section{CASO CLÍNICO}

Paciente femenina, de 80 años de edad, quien consulta por xerostomía y xeroftalmia, de larga data. Al examen físico como datos positivos: inyección conjuntival, pobre lago salival. Laboratorio: anticuerpos antinucleares ANAS y Anti Ro positivos.
TABLA No 1. CRITERIOS CLASIFICATORIOS DEL SINDROME DE SJOGREN: CONSENSO EUROPEO AMERICANO 2002.

1. Síntomas orales (una respuesta positiva)

1.1. Sensación de boca seca por un período superior a tres meses

1.2. Inflamación de glándula salival recurrente 1.3. Necesidad constante de beber líquidos

2. Síntomas oculares (una respuesta positiva)

2.1. Sensación de ojos secos por un período superior a tres meses

2.2. Sensación de arenilla ocular recurrente

2.3. Utilizar lágrimas artificiales más de tres veces al día

3. Signos oculares (una prueba positiva)

3.1. Prueba de Schirmer sin anestesia inferior o igual a $5 \mathrm{~mm}$ a los 5 minutos

3.2. Puntuación de 4 o más (escala de Bijsterveld) en la tinción con rosa de Bengala

4. Alteración de las glándulas salivales (una prueba positiva)

4.1. Gammagrafía de glándulas salivales con retardo

de captación, hipo concentración y/o retardo de

excreción.

4.2. Sialografía con alteraciones difusas ductales y acinares

4.3. Flujo salival sin estimular de $1,5 \mathrm{ml}$ o menos en 15 minutos

5. Histopatología

Biopsia salival grado 3-4 de la clasificación de Chisholm y Mason

6. Inmunología (una prueba positiva)

6.1. Anti-Ro/SS-A

6.2. Anti-La/SS-B

Se realiza entonces, gammagrafía de glándulas salivales, después de la administración de $15 \mathrm{mCi}$ ( $555 \mathrm{MBq}$ ) de 99mTc-pertecneciato por vía endovenosa, en la posición de decúbito supino, proyección anterior de cabeza, en gammacámara Adac, Genesys Epic Single Head, con colimador de baja energía y alta resolución; siendo obtenidas imágenes a cada segundo inicialmente durante el primer minuto, y luego cada 15 segundos, durante 30 minutos. Se realiza estímulo cítrico (jugo de limón), a los 15 minutos de iniciado el procedimiento e imágenes estáticas posteriores en las proyecciones anterior, lateral derecha y lateral izquierda. Estas imágenes son procesadas en computador Pegasys Epic, diseñándose áreas de interés sobre las glándulas parótidas y submaxilares y confeccionando curvas de tiempo/actividad de cada glándula.

Para su interpretación se evalúa la captación por las glándulas salivales, concentración y 
la excreción espontánea y la respuesta al estimulo acido.

Al estudio gammagráfico se observa severo compromiso de la concentración del trazador por las glándulas salivares, que apenas se esbozan durante la fase parenquimatosa, sin evidencia de eliminación del radioelemento durante la fase excretora (Figuras No 1 y No 2).

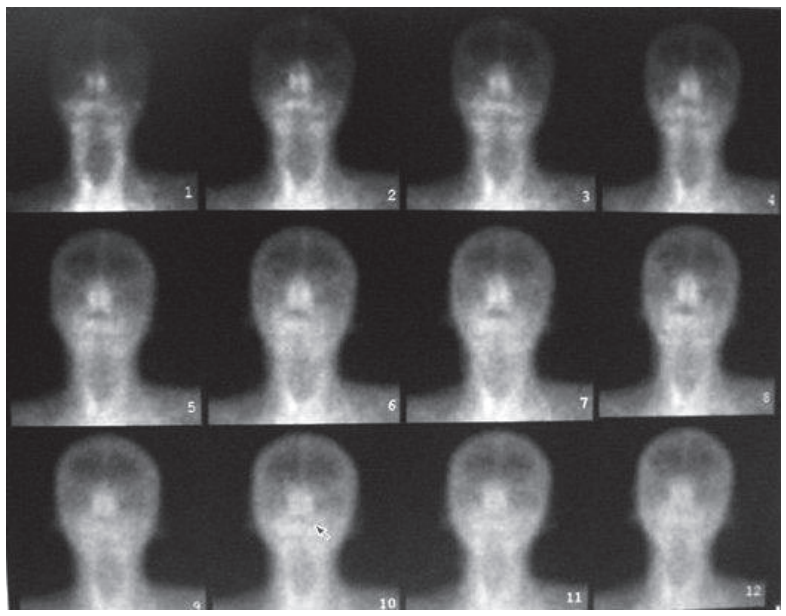

Figura No 1. Estudio dinámico cada 2,5 minutos, durante 30 minutos, después de la administración endovenosa del trazador, con estimulo cítrico a los 15 minutos, en la proyección anterior, donde se observa severo compromiso de la captación del trazador por las glándulas salivares que apenas se esbozan.

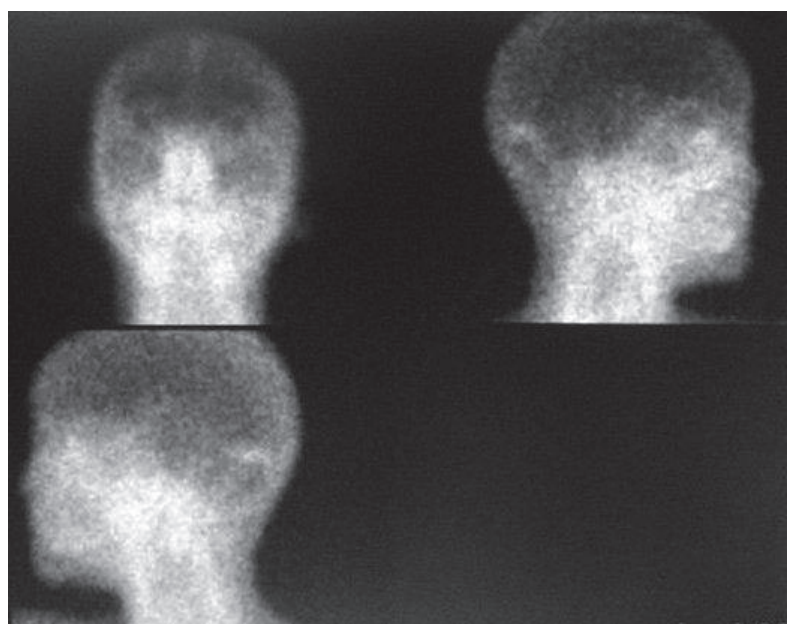

Figura No 2. Imágenes estáticas a los 45 minutos, en las proyecciones anterior, lateral derecha y lateral izquierda, notando hipo concentración marcada por las glándulas salivales y muy poca actividad en boca.

La Figura No 3 muestra el diseño de las áreas de interés que se realizaron sobre las glándulas parótidas y submaxilares, en cuanto que las Figuras No 4 y No 5 presentan las curvas tiempo / actividad generadas por ese análisis, que al ser aplanadas confirman los hallazgos ya descritos.

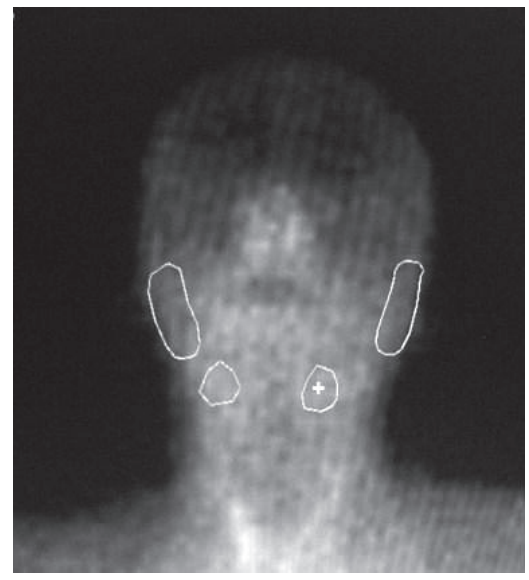

Figura No 3. Imagen con las áreas de interés sobre las glándulas parótidas y submaxilares.

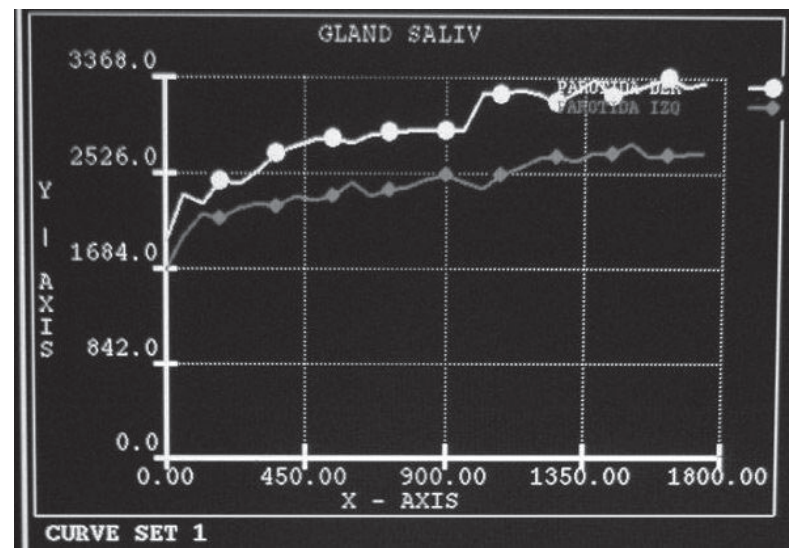

Figura No 4. Curvas aplanadas anormales de tiempo/ actividad de las glándulas parótida derecha (línea superior) y parótida izquierda (línea inferior).

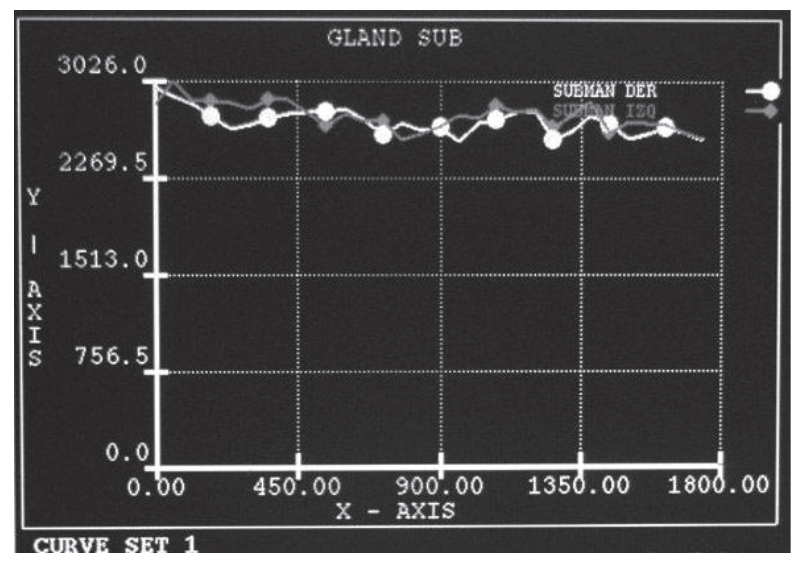

Figura No 5. Curvas anormales de tiempo/actividad de las glándulas submaxilares derecha (línea inferior) e izquierda (línea superior) 
Betancourt Piñeres Aiken Felipe, Milanés Pérez Rosa Isabel, Bonnet Palencia Igor Iván, Padilla Tovar Lacides,

Moreno Grau Álvaro, Álvarez Romero Erick, Muñoz Méndez José Thomas

La biopsia de glándula salival menor (labio inferior), reveló mucosa labial constituida por seis lóbulos de acinos seromucosos, algunos atróficos rodeados de múltiples focos de infiltrado inflamatorio crónico linfoplasmocitario constituido por más de cincuenta células y hallazgos histopatológicos consistentes con el Síndrome de Sjögren.

Se confirma entonces el diagnóstico de Síndrome de Sjögren primario, siendo manejado con cloroquina una tableta diaria y controles periódicos, con evolución satisfactoria.

El presente caso cumple con todos los criterios diagnósticos del Consenso Europeo Americano del año 2002. En conjunto los hallazgos gammagráficos mencionados, la biopsia de labio inferior y demás criterios clasificatorios de este síndrome, permitieron realizar un diagnóstico definitivo de Síndrome de Sjögren.

\section{DISCUSIÓN}

El Síndrome de Sjögren es más frecuente en mujeres de mediana edad, en una relación mujer-hombre de 9:1 (3,4). Los síntomas clínicos principales y las complicaciones están relacionados con la destrucción de las glándulas y la sequedad de las mucosas. Es una enfermedad sistémica autoinmune, crónica, inflamatoria caracterizada por la infiltración de las glándulas exocrinas por linfocitos y células plasmáticas $(5,6)$.

El carácter sindrómico del Síndrome de Sjögren primario y su tendencia a la evolución crónica dificultan su diagnóstico en el momento en que consulta el paciente, ya que el resultado de las distintas pruebas diagnósticas varía en función del tiempo de evolución de la entidad.

No cabe duda que la estrategia diagnóstica del Síndrome de Sjögren se basa en el estudio de los componentes ocular y bucal. Ante la sospecha de un síndrome de Sjögren una medida diagnóstica adecuada sería practicar primero la tinción con rosa de Bengala para demostrar la xeroftalmia, posteriormente la gammagrafía salival y en último término la biopsia.
Sin embargo, las diversas pruebas diagnósticas utilizadas presentan cifras variables de sensibilidad y especificidad, cuando son analizadas individualmente, hechos que ponen de manifiesto la dificultad que existe en ocasiones para diagnosticar el cuadro.

Destacamos entre los estudios propuestos la gammagrafía de glándulas salivares que permite la evaluación morfo funcional de las parótidas y submaxilares. El radionúclido utilizado es el 99mTc-pertecneciato, que se administra por vía endovenosa, es captado y excretado por las glándulas salivales.

El Síndrome de Sjôgren puede mostrar patrones gammagráficos diversos según su estadío; ya que en las fases iniciales se observa más comúnmente asimetría en la captación del trazador en las parótidas, así como mayor compromiso relativo de las submaxilares, en las fases tardías, como el caso estudiado, donde se observa compromiso funcional panglandular (9).

De otra parte, algunos autores consideran que el estudio radio nuclear ofrece información pronostica de los pacientes acometidos por Síndrome de Sjôgren primario, ya que aquellos que muestran hipocaptaciones más severas del radiotrazador generalmente presentan mayor posibilidad de compromiso sistémico y autoinmune, mayor incidencia de linfoma y menor sobrevida (10).

En el caso clínico presentado, se realizó todo el proceso para el diagnóstico definitivo de Síndrome de Sjögren, en el cual fue de vital importancia la evaluación sindromática del paciente, complementado con la evaluación gammagráfíca de las glándulas salivales; estudio que mostró un severo compromiso funcional de las glándulas salivares mayores. Cabe destacar que aunque en la ancianidad se puede observar una disminución fisiológica en la actividad de las glándulas salivares, las curvas tiempo /actividad generalmente mantienen su patrón característico.

El tratamiento del Síndrome de Sjógren es sintomático, utilizando los recursos a disposición para aliviar la xeroftalmía, así como la xerostomía, que incluyen la aplicación de 
lagrimas artificiales, ungüentos lubricantes y cloroquina; si no hay mejoría en cuanto a la producción de lagrimas y saliva se recomienda pilocarpina en dosis diarias de $15 \mathrm{mgs}$. diarios. En las manifestaciones extra glandulares y en las formas severas refractarias, a las medidas generales y a el tratamiento conservador, se le agregan los inhibidores del factor de necrosis tumoral alfa, sin embargo se necesitan más estudios para evaluar su efectividad.

Entre las medidas generales hay que resaltar una buena higiene bucal, visitar al odontólogo para prevenir las caries y pérdida de los dientes, sustitutos de saliva, entre otros, e igualmente, evitar medicamentos que produzcan resequedad bucal como los antihistamínicos o descongestionantes y evitar el alcohol.

Esta enfermedad cursa con gran morbilidad por la resequedad ocular y bucal, con sobreinfección agregada. El pronóstico depende de otras enfermedades presentes asociadas y dentro de las complicaciones se mencionan el linfoma, la infección pulmonar, la insuficiencia renal y las vasculitis entre otras, siendo estas dos últimas las menos comunes.

\section{CONCLUSIÓN}

El presente caso permite mostrar la utilidad de la gammagrafía de glándulas salivares, un método diagnóstico no invasivo y su correlación con la biopsia de glándulas salivales menores en el Síndrome de Sjögren, patología multifactorial de difícil diagnóstico, sobre todo en sus fases iníciales, donde la clínica, así como los estudios paraclínicos juegan papel primordial para su confirmación.

CONFLICTOS DE INTERÉS: ninguno que declarar.

FINANCIACIÓN: recursos propios de los autores. Estudios paraclínicos e insumos aportados dentro la atención asistencial.

\section{REFERENCIAS BIBLIOGRÁFICAS}

1. Díaz-Rubio, M., Espinos D. Tratado de Medicina Interna. Madrid: editorial Médica Panamericana, 1994: Tomo 2: 2825-2827.

2. Baum BJ, Fox CF, Neuman RD. The Salivary Glands. En: Harbert J, Eckelman W, Neuman R, de. Nuclear Medicine: Diagnosis and Therapy. New York: Thieme Medical Publishers, Inc, 1996: 439-44.

3. Vivino, F. B.; Al-Hashimi, I.; Khan, Z.; LeVeque, F. G.; Salisbury, P. L.; Tran-Johnson, T. K.; Muscoplat, C. C.; Trivedi, M.; Goldlust, B. \& Gallagher, S. C. Pilocarpine Tablets for the Treatment of Dry Mouth and Dry Eye Symptoms in Patients With Sjogren Syndrome. Arch. Intern. Med., 1999; 159:174-81.

4. Saito T, Fukuda H, Horikawa M, Ohmori K, Shindoh M, Amemiya A. Salivary gland scintigraphy with 99mTc-pertechnetate in Sjogren 's syndrome: relationship to clinicopathologic features of salivary and lacrimal glands. J Oral Pathol Med, 1997; 26: 46-50..

5. Hughes PM, Carson K, Hill J, Hastings D. Scintigraphic evaluation of sialadenitis. Br. J. Radiol, 1994; 67: 328-31.

6. Marton, K.; Boros, I.; Fejerdy, P. \& Madlena, M. Evaluation of unstimulated flow rates of whole and palatal saliva in healthy patients wearing complete dentures and in patients with Sjogren's syndrome. J.Prosthet. Dent., 2004; 91:577-81.

7. Binon, P. P. Thirteen-year follow-up of a mandibular implant-supported fixed complete denture in a patient with Sjogren's syndrome: a clinical report. J. Prosthet. Dent., 2005; 94:409-13.

8. Vitali C, Bombardieri S, Jonsson R, Moutsopoulos HM, Alexander EL, Carsons SE, et al. Classification criteria for Sjogren's syndrome: a revised version of the European criteria proposed by the American-European Consensus Group. Ann Rheum Dis. 2002;61:554-8.

9. Güne SE, Yilmaz S, Karalezli A, Aktas A. Quantitative and visual evaluation of salivary and thyroid glands in patients with primary Sjögren's syndrome using salivary gland scintigraphy: relationship with clinicopathological features of salivary, lacrimal and thyroid glads. Nucl Med Commun. 2010; 31(7):666-72.

10. Ramos-Casals M, Brito-Zerón P, Pérez-DE-Lis M, Díaz-Lagares $C$, Bove $A$, Soto MJ, Jiménez I, Belenguer R, Siso A, Muxí A, Pons F. Clinical and prognostic significance of parotid scintigraphy in 405 patients with primary Sjörgen's syndrome. J Rheumatol. 2010; 37(3):585-90. 